\title{
Self-reinforcing directionality generates truncated Lévy walks without the power-law assumption
}

DOI:

10.1103/PhysRevE.103.022132

\section{Document Version}

Accepted author manuscript

Link to publication record in Manchester Research Explorer

\section{Citation for published version (APA):}

Fedotov, S., Han, D., \& Korabel, N. (2021). Self-reinforcing directionality generates truncated Lévy walks without the power-law assumption. Physical Review E: covering statistical, nonlinear, biological, and soft matter physics, 103, [022132]. https://doi.org/10.1103/PhysRevE.103.022132

\section{Published in:}

Physical Review E: covering statistical, nonlinear, biological, and soft matter physics

\section{Citing this paper}

Please note that where the full-text provided on Manchester Research Explorer is the Author Accepted Manuscript or Proof version this may differ from the final Published version. If citing, it is advised that you check and use the publisher's definitive version.

\section{General rights}

Copyright and moral rights for the publications made accessible in the Research Explorer are retained by the authors and/or other copyright owners and it is a condition of accessing publications that users recognise and abide by the legal requirements associated with these rights.

\section{Takedown policy}

If you believe that this document breaches copyright please refer to the University of Manchester's Takedown Procedures [http://man.ac.uk/04Y6Bo] or contact uml.scholarlycommunications@manchester.ac.uk providing relevant details, so we can investigate your claim.

\section{OPEN ACCESS}




\title{
Self-reinforcing directionality generates truncated Lévy walks without the power-law assumption
}

\author{
Daniel Han, ${ }^{1}$ Marco A. A. da Silva, ${ }^{2}$ Nickolay Korabel, ${ }^{1}$ and Sergei Fedotov ${ }^{1}$ \\ ${ }^{1}$ Department of Mathematics, University of Manchester, M13 9PL, UK \\ ${ }^{2}$ Faculdade de Ciências Farmacêuticas de Ribeirão Preto, \\ Universidade de São Paulo (FCFRP-USP), Ribeirão Preto, Brazil
}

(Dated: March 3, 2021)

\begin{abstract}
We introduce a persistent random walk model with finite velocity and self-reinforcing directionality, which explains how exponentially distributed runs self-organize into truncated Lévy walks observed in active intracellular transport by Chen et. al. [Nat. mat., 2015]. We derive the nonhomogeneous in space and time, hyperbolic PDE for the probability density function (PDF) of particle position. This PDF exhibits a bimodal density (aggregation phenomena) in the superdiffusive regime, which is not observed in classical linear hyperbolic and Lévy walk models. We find the exact solutions for the first and second moments and criteria for the transition to superdiffusion.
\end{abstract}

\section{INTRODUCTION}

Transport in biology is crucial on multiple scales to maintain life and deviations from normal movement are hallmarks of disease and ageing $[1,2]$. From organisms to sub-cellular molecules, their motion is usually described by persistent random walks with finite velocities (run and tumble models) [3-7]. The preference for these velocity jump models over space jump random walks arise due to the physical constraints of organisms not instantaneously jumping in space and an inertial resistance to changes in direction. In recent years, Lévy walks [8] attracted attention in modelling movement patterns of living things [9], from sub-cellular [10-12] to organism [13-15] scales. Until now, Lévy walks have been mostly described by coupled continuous time random walks (CTRW) [8], various fractional PDEs [16-22] and integro-differential equations $[23,24]$. These approaches require power-law distributed running times with divergent first and second moments as an ab initio assumption. However, in many cases this assumption is difficult to justify, leading to ongoing discussions about the origin of power-law distributed runs, the Levy walk observed in nature $[9,25]$ and Lévy foraging hypothesis [26, 27].

Experiments exhibiting Lévy-like motion cannot be modeled with pure power-law jump or running time distributions due to finite limits in physical systems [28]. So theoretically, power-law distributions are often truncated [29] or exponentially tempered [30]. Specifically for active cargo transport in cells, it was discovered that the motion is composed of multiple short runs that self-organize into longer, truncated power-law distributed, uni-directional flights (truncated Lévy walks) [10]. Explanations for this phenomenon have been attempted in terms of selfreinforcing directionality generated by co-operative motor protein transport [10]. Yet, the question remains, can a persistent random walk model generate superdiffusion without power-law distributed runs through the self-organization of exponentially distributed runs?

In this paper, we propose a new, spatially and temporally inhomogeneous model that generates exponentially truncated Lévy walks through self-reinforced directionality without the standard assumption of power-law distributed runs. This exponential truncation is not directly introduced but rather arises naturally from the same microscopic mechanism that generates the power-law distribution. Finally, simulated densities of uni-directional flight lengths from this model show excellent agreement with truncated power-law distributions observed in experiments [10]. It is worth noting that there are a few examples where the power-law assumption has not been used as a starting point: superdiffusion of ultracold atoms [31] and a random walk driven by an ergodic Markov process with switching [32].

\section{SELF-REINFORCING DIRECTIONALITY}

Consider a particle moving to the right and left in $1 \mathrm{D}$ with constant speed $\nu$ and exponentially distributed running time with rate $\lambda$. In the standard alternating case, the particle would change directions with probability 1 . To consider the instance when there is a probability that the particle continues in the same direction as the previous run, we introduce the transition probability matrix [33]:

$$
\boldsymbol{Q}=\left[\begin{array}{cc}
q_{+} & 1-q_{+} \\
1-q_{-} & q_{-}
\end{array}\right]
$$

where $q_{+}$is the conditional transition probability that the particle will continue in the positive direction given it was moving in the positive direction before. Similarly, $q_{-}$corresponds to the particle moving in the negative direction. The standard alternating case corresponds to $q_{+}=q_{-}=0$.

In order to model self-reinforcing directionality using the matrix $\boldsymbol{Q}$, we introduce relative times, $t^{+} / t$ and $t^{-} / t$, that the particle moves in the positive and negative direction during time $t$. The key point is to define the 
probabilities in (1) as

$$
q_{ \pm}=w \frac{t^{ \pm}}{t}+(1-w) \frac{t^{\mp}}{t} .
$$

Here we introduce the persistence probability, $w$, which parameterizes the extent that changes of direction are affected by relative times. If $w=1 / 2$ then the transition probabilities in both directions are the same: $q_{+}=$ $q_{-}=1 / 2$. If $w>1 / 2$ then the matrix $\boldsymbol{Q}$ has repetition compulsion properties: the longer a particle spends moving in the one direction, the greater the probability to maintain directionality. In other words, the particle has a tendency to repeat its past behavior. Not only does $q_{ \pm}$depend on the previous run, but it depends on the entire history of run directions and run times. Figure 1 illustrates self-organization of individual runs into long uni-directional flights.

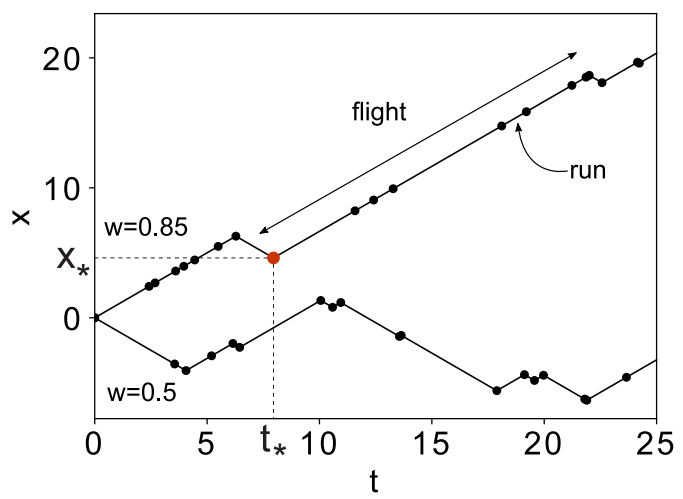

FIG. 1. Two realizations of the random walk with selfreinforcing directionality using the transition matrix (1) with $q_{ \pm}(x, t)$ from (2). The continuous trajectories (black solid lines) are labeled according to the persistence probability $w=0.85$ and $w=0.5$. The black dots show the beginning and end of individual exponentially distributed runs. A flight and run are annotated. The parameters are $\nu=1$ and $\lambda=1$. The trajectory with $w=0.85$ has an initial velocity $\nu$ and $w=0.5,-\nu$. The enlarged red dot shows the beginning of a uni-directional flight at $x_{*}$ and $t_{*}$.

Let us show that the conditional transition probabilities (2) can be expressed in terms of particle position $x=x_{0}+\nu\left[t^{+}-t^{-}\right]$. Since $t=t^{+}+t^{-}$, the relative times can be written as

$$
\frac{t^{ \pm}}{t}=\frac{1}{2}\left(1 \pm \frac{x-x_{0}}{\nu t}\right)
$$

Then substituting (3) into (2), we find that the probabilities $q_{+}$and $q_{-}$depend on $x$ and $t$,

$$
q_{ \pm}(x, t)=\frac{1}{2}\left[1 \pm \alpha \frac{x-x_{0}}{\nu t}\right] \text { with } \alpha=2 w-1 .
$$

Since our random walker is moving with finite speed $\nu$, the relation $\left(x-x_{0}\right) / \nu t \leq 1$ must hold. Once again, the formula (4) shows self-reinforcing directionality since if $\alpha>0(w>1 / 2)$ then the transition probability $q_{+}(x, t)$ is an increasing function of particle position $x$ and the opposite for $q_{-}(x, t)$. Here we should emphasize that the probabilities $q_{ \pm}$depend not only on the direction of the previous run, but on the full history of run directions which determine its current position, $x$. So $x>x_{0}$ means that the positively directed runs outlived those in the negative direction. That is why this can be referred to as self-reinforcing directionality. As we will show below, transition probabilities (4) give us a new methodology to model truncated Lévy walk motion, without the standard requirement of power-law distributed runs.

The equations for probability density functions (PDFs) of particles moving right $(+)$ and left $(-), p_{ \pm}(x, t)$, can be written in terms of transition matrix $\boldsymbol{Q}$ as

$$
\begin{aligned}
{\left[\begin{array}{l}
p_{+}(x, t+\Delta t) \\
p_{-}(x, t+\Delta t)
\end{array}\right] } & =(1-\lambda \Delta t)\left[\begin{array}{l}
p_{+}(x-\nu \Delta t, t) \\
p_{-}(x+\nu \Delta t, t)
\end{array}\right] \\
& +\boldsymbol{Q}^{T}\left[\begin{array}{l}
p_{+}(x, t) \\
p_{-}(x, t)
\end{array}\right] \lambda \Delta t .
\end{aligned}
$$

Rearranging and taking the limit $\Delta t \rightarrow 0$, we can write the equations for $p_{ \pm}(x, t)$ as

$$
\frac{\partial p_{ \pm}}{\partial t} \pm \nu \frac{\partial p_{ \pm}}{\partial x}=-\lambda\left(1-q_{ \pm}(x, t)\right) p_{ \pm}+\lambda\left(1-q_{\mp}(x, t)\right) p_{\mp} .
$$

Note these equations (6) can be rewritten in terms of space and time dependent switching rates

$$
\lambda_{ \pm}(x, t)=\lambda\left(1-q_{ \pm}(x, t)\right) .
$$

These switching rates will be used to show how the exponentially truncated power-law distribution of flights arise from exponentially distributed runs. Defining total density and flux as

$$
p(x, t)=p_{+}+p_{-}, \quad J(x, t)=\nu p_{+}-\nu p_{-}
$$

and using standard methods $[6,7,34]$ with (4), we can obtain the system of equations,

$$
\frac{\partial p}{\partial t}=-\frac{\partial J}{\partial x}, \quad \frac{\partial J}{\partial t}=-\nu^{2} \frac{\partial p}{\partial x}-\lambda\left(J-\frac{\alpha\left(x-x_{0}\right)}{t} p\right) .
$$

The initial conditions are

$$
p(x, 0)=\delta\left(x-x_{0}\right), \quad J(x, 0)=\nu(2 u-1) \delta\left(x-x_{0}\right),
$$

where $u \in[0,1]$ is the probability that the intial velocity is $\nu$. Finally from (8), we can find the hyperbolic PDE with a non-homogeneous in space and time advection term

$$
\frac{\partial^{2} p}{\partial t^{2}}+\lambda \frac{\partial p}{\partial t}=\nu^{2} \frac{\partial^{2} p}{\partial x^{2}}-\frac{\lambda \alpha}{t} \frac{\partial\left(\left(x-x_{0}\right) p\right)}{\partial x}, t>0 .
$$

The advection term of Eq. (10) is unconventional because it depends on the initial position $x_{0}$. Furthermore, if the initial conditions are symmetric, $u=1 / 2$ (see (9)), 
then the average drift is zero. Clearly, (10) is a modification of the classical telegraph or Cattaneo equation $[7,34,35]$, with a time and space dependent advection term. In what follows, we will show that this additional term generates superdiffusion. In fact, a generalized Cattaneo equation generating superdiffusion has been formulated using the Riemann-Liouville fractional derivative [16]. The advantage of Eq. (10) over fractional PDEs is that it is far simpler and does not require integral operators in time. To the authors' knowledge, the hyperbolic PDE (10) is the first formulation of truncated Lévy walks without integral operators [23]. In the diffusive limit, when $\lambda \rightarrow \infty$ and $\nu \rightarrow \infty$ such that $\nu^{2} / \lambda$ is a constant, (10) becomes the governing advection-diffusion equation for the continuous approximation of the elephant random walk [36].

It is interesting to note that the system of equations (6) with transition rates (4) can be mapped to the hyperbolic model for chemotaxis [37] with an unorthodox external stimulus $S(x, t)$ obeying the Hamilton-Jacobi equation for a free particle. In terms of effective turning rates, $\lambda_{ \pm}$ introduced in (7), that depend on the gradient of external stimulus:

$$
\lambda_{ \pm}\left(S_{x}\right)=\frac{\lambda}{2}\left[1 \mp \frac{1}{\nu} S_{x}\right] \text {, with } S(x, t)=\frac{\alpha\left(x-x_{0}\right)^{2}}{2 t} .
$$

Then the Hamilton-Jacobi equation for the external stimulus is

$$
\frac{\partial S}{\partial t}+\frac{1}{2 \alpha}\left(\frac{\partial S}{\partial x}\right)^{2}=0
$$

This provides insight into how the external stimulus, $S$, generates superdiffusion rather than the conventional ballistic motion in chemotaxis.

Monte Carlo simulations of the random walk corresponding to (10) is as follows:

1. Set initial conditions $x_{0}=0$ and $t_{0}=0$. For initial velocity draw a uniformly distributed random number $U \in[0,1)$, if $U<u$ then $v_{0}=\nu$ and otherwise $v_{0}=-\nu$.

2. Generate an exponentially distributed random time $T_{0}=-1 / \lambda \log (1-V)$ where $V$ is a uniformly distributed random number in $[0,1)$.

3. Update position and time to $x_{1}=x_{0}+v_{0} T_{0}, t_{1}=$ $t_{0}+T_{0}$ respectively. For updating velocity, draw a uniformly distributed random number $W \in[0,1)$, then

(a) If $v_{0}=\nu$ and $W<q_{-}\left(x_{1}, t_{1}\right)=1 / 2-$ $\alpha\left(x_{1} / 2 \nu t_{1}\right)$ then $v_{1}=-\nu$.

(b) If $v_{0}=-\nu$ and $W<q_{+}\left(x_{1}, t_{1}\right)=1 / 2+$ $\alpha\left(x_{1} / 2 \nu t_{1}\right)$ then $v_{1}=\nu$.

4. Repeat steps 2 and 3 until $t_{n}=t_{0}+\sum_{i=0}^{n-1} T_{i}$ reaches the end of the simulation time $t_{\text {end }}$.

\section{MOMENTS AND SUPERDIFFUSION}

Now, we show that the variance for the underlying random process, $x(t)$, exhibits superdiffusive behavior: $\operatorname{Var}\{x(t)\} \propto t^{2 \alpha}$ with $1 / 2<\alpha<1$. The moments of random walk position,

$$
\mu_{n}(t)=\int_{-\infty}^{\infty} x^{n} p(x, t) d x
$$

can be found from (10) as

$$
\frac{d^{2} \mu_{n}}{d t^{2}}-\nu^{2} n(n-1) \mu_{n-2}+\lambda \frac{d \mu_{n}}{d t}-\frac{\lambda \alpha n}{t} \mu_{n}=0 .
$$

Taking the Laplace transform and solving the resulting differential equations for the first and second moments using the initial conditions,

$$
\begin{gathered}
\mu_{1}(0)=\mu_{2}(0)=0 \\
\frac{d \mu_{1}(0)}{d t}=\nu(2 u-1), \frac{d \mu_{2}(0)}{d t}=0,
\end{gathered}
$$

derived from (9), we can obtain

$$
\begin{aligned}
& \hat{\mu}_{1}(s)=\nu(2 u-1) s^{-1-\alpha}(s+\lambda)^{\alpha-1}, \\
& \hat{\mu}_{2}(s)=\frac{2 \nu^{2}}{\lambda(2 \alpha-1)}\left[s^{-1-2 \alpha}(s+\lambda)^{2 \alpha-1}-s^{-2}\right]
\end{aligned}
$$

The inverse Laplace transform gives

$$
\begin{aligned}
& \mu_{1}(t)=\nu(2 u-1) t_{1} F_{1}(1-\alpha, 2,-\lambda t), \\
& \mu_{2}(t)=\frac{2 \nu^{2} t}{\lambda(2 \alpha-1)}\left[{ }_{1} F_{1}(1-2 \alpha, 2,-\lambda t)-1\right]
\end{aligned}
$$

where ${ }_{1} F_{1}(a, b, z)$ is the Kummer confluent hypergeometric function. In the long time limit $(s \rightarrow 0)$, we obtain

$$
\mu_{1}(t) \simeq \frac{\nu \lambda^{\alpha-1}(2 u-1)}{\Gamma(\alpha+1)} t^{\alpha}
$$

and

$$
\mu_{2}(t) \simeq \begin{cases}\frac{2 \nu^{2}}{\lambda(1-2 \alpha)} t, & -1<\alpha<1 / 2 \\ \frac{2 \nu^{2} \lambda^{2 \alpha-2}}{(2 \alpha-1) \Gamma(2 \alpha+1)} t^{2 \alpha}, & 1 / 2<\alpha<1\end{cases}
$$

Clearly, the random walk exhibits superdiffusive behavior for $1 / 2<\alpha<1$. The variance, $\operatorname{Var}\{x(t)\}=\mu_{2}(t)-$ $\mu_{1}(t)^{2}$, is

$$
\operatorname{Var}\{x(t)\} \simeq \begin{cases}\frac{2 \nu^{2}}{\lambda(1-2 \alpha)} t, & -1<\alpha<1 / 2 \\ A \nu^{2} \lambda^{2 \alpha-2} t^{2 \alpha}, & 1 / 2<\alpha<1\end{cases}
$$

where $A=\frac{2}{(2 \alpha-1) \Gamma(2 \alpha+1)}-\frac{(2 u-1)^{2}}{\Gamma^{2}(\alpha+1)}$. Fig. 2 shows that results of Monte Carlo simulations are in perfect agreement with the analytical solution of the second moment. This clearly demonstrates the emergence of superdiffusion since for $w<3 / 4(\alpha<1 / 2), \mu_{2}(t)=\left\langle x^{2}(t)\right\rangle \propto t$, whereas $\left\langle x^{2}(t)\right\rangle \propto t^{2 \alpha}$ for $w>3 / 4(\alpha>1 / 2)$. 


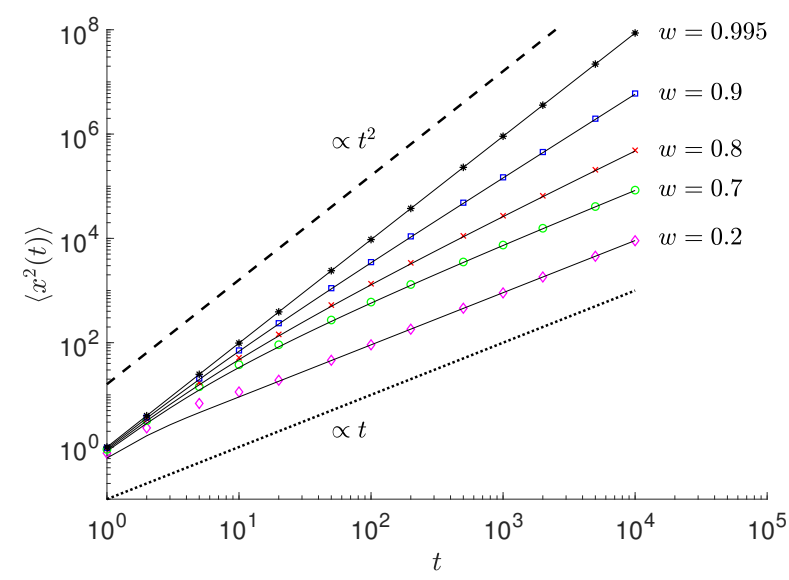

FIG. 2. Mean squared displacements, $\left\langle x^{2}(t)\right\rangle=\mu_{2}(t)$, from simulated trajectories (data points) compared with the analytical solutions (solid lines). Each pair is annotated with the simulation value of $w$. Each simulation, contained $N=10^{5}$ particles that ran for a simulation time $t=10^{4}$ with parameters $u=1, \nu=1$ and $\lambda=1$. Diffusion (dotted line), $\left\langle x^{2}(t)\right\rangle \sim t$, and ballistic motion (dashed line), $\left\langle x^{2}(t)\right\rangle \sim t^{2}$, are also shown.

\section{CONDITIONAL TRUNCATED POWER-LAW FLIGHT DISTRIBUTION}

In what follows, we show analytically how selfreinforcing directionality generates a truncated Lévy walk with exponentially tempered power-law distributed flights. Let us find the conditional distribution of flights given the particle changes direction at the position $x_{*}$ and time $t_{*}$ (illustrated in Fig. 1 where the particle changes velocity from $-\nu$ to $\nu$ ). First, we calculate the conditional survival function, $\Psi_{ \pm}\left(\tau \mid x_{*}, t_{*}\right)$, for flights moving in the positive $(+)$ and negative $(-)$ direction. This function gives us the probability that the random duration of a flight, $T$, is greater than $\tau$. The rates, $\lambda_{ \pm}$ (7) define the conditional switching rate along the particle trajectory starting at position $x_{*}$ and time $t_{*}$ as $\lambda_{ \pm}\left(\tau \mid x_{*}, t_{*}\right)=\lambda\left(1-q_{ \pm}\left(x_{*} \pm \nu \tau, t_{*}+\tau\right)\right)$. If we rearrange using $\alpha=2 w-1$, then

$$
\lambda_{ \pm}\left(\tau \mid x_{*}, t_{*}\right)=\lambda(1-w)+\frac{\gamma_{ \pm}}{t_{*}+\tau}
$$

where

$$
\gamma_{ \pm}=\left(w-\frac{1}{2}\right) \lambda\left(t_{*} \mp \frac{x_{*}}{\nu}\right)
$$

Using this, we can find the conditional survival function by

$$
\Psi_{ \pm}\left(\tau \mid x_{*}, t_{*}\right)=\exp \left(-\int_{0}^{\tau} \lambda_{ \pm}\left(s \mid x_{*}, t_{*}\right) d s\right)
$$

Then, we can see that the constant term in (18) produces an exponential tempering factor and the term inversely proportional to the running time, $\tau$, generates a powerlaw with exponent $\gamma_{ \pm}$. Explicitly, we can write

$$
\Psi_{ \pm}\left(\tau \mid x_{*}, t_{*}\right)=e^{-(1-w) \lambda \tau}\left(\frac{t_{*}}{t_{*}+\tau}\right)^{\gamma_{ \pm}} .
$$

The conditional distribution of flight lengths $l=\nu \tau$ is given by: $F_{ \pm}\left(l \mid x_{*}, t_{*}\right)=1-\Psi_{ \pm}\left(l / \nu \mid x_{*}, t_{*}\right)$.

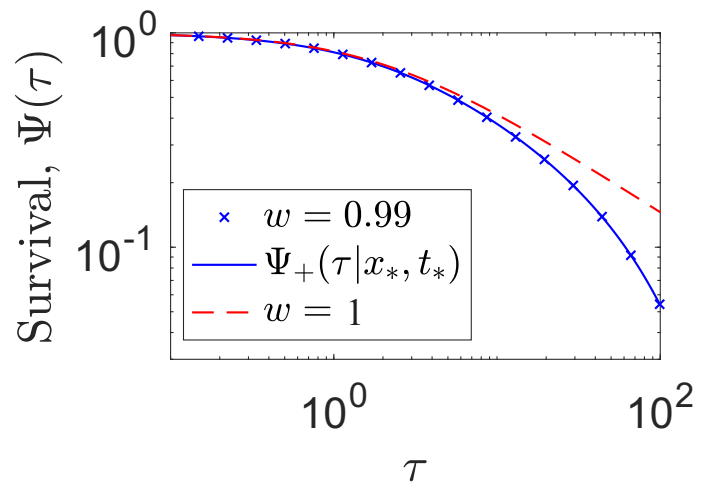

FIG. 3. The survival function $\Psi(\tau)$ of unidirectional flight running times $\tau$, estimated using the non-parametric KaplanMeier method, from the simulation of $N=2 \times 10^{6}$ unidirectional flights with $w=0.99, v=1, \lambda=1, t_{*}=2$ and $x_{*}=1$. The conditional survival function of flights from numerical simulations (blue crosses) and the analytical truncated powerlaw conditional survival function $\Psi_{+}\left(\tau \mid x_{*}, t_{*}\right)$ from (19) (blue line) show excellent correspondence. The survival function of $\Psi_{+}\left(\tau \mid x_{*}, t_{*}\right)$ without the exponential truncation, $w=1$, is shown for comparisons (red dashed line).

Fig. 3 shows excellent correspondence between numerical simulations and the analytical formula (19) for $\Psi_{+}\left(\tau \mid x_{*}, t_{*}\right)$.

Usually exponential truncation (tempering) is introduced by simply multiplying the power-law jump or waiting time densities by an exponential factor with an additional parameter, leading to tempered fractional calculus [30]. The advantage of our model is that both exponential tempering and power-law flight distribution are generated through a single microscopic mechanism involving self-reinforced directionality and the subsequent analysis of (10) is more convenient than tempered fractional calculus. An interesting feature of our model is that despite the exponential truncation of flights, the variance (17) still exhibits superdiffusive behavior for $1 / 2<\alpha<1$ $(3 / 4<w<1)$.

Finally, we can obtain numerically the $\mathrm{PDF}, p(l)$, of flight length, $l$, without the condition that flights begin at $x_{*}$ and $t_{*}$. In the case of strong directionality as $w \rightarrow 1, p(l)$ should approach a pure power-law since the truncation length $\nu / \lambda(1-w) \rightarrow \infty$. Figure 4 confirms this and shows that, when $w=0.999$, a power-law density is recovered for more than two orders of magnitude. Next, we provide evidence that the truncated power-law PDF shows excellent correspondence to published data on uni-directional endosome flights. 


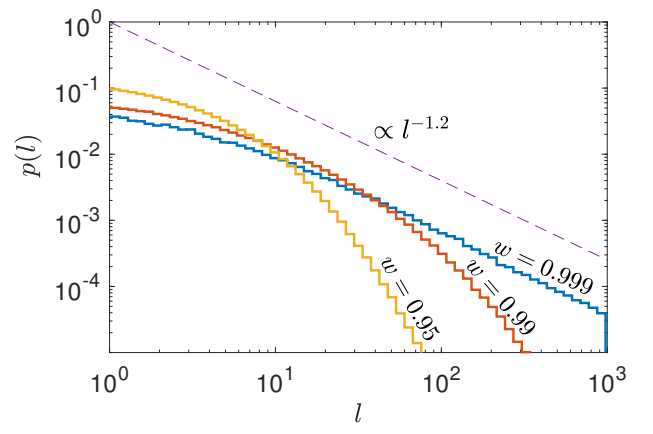

FIG. 4. The PDFs $p(l)$ (solid lines) of unidirectional flight lengths $l$ from the simulations of $N=10^{5}$ particles with varying $w, t=10^{3}, v=1$ and $\lambda=1$.

\section{EXPERIMENTAL EVIDENCE}

Truncated Lévy walk behavior is observed experimentally in intracellular transport [10], which arises from the self-organization of exponentially distributed runs, $x_{i}$, into uni-directional flights, $x_{f}[10]$. Here, we switch from $l$ to $x_{f}$ to avoid confusion between theoretical and experimental flights. Until now, there had been no governing PDE like (10) and an underlying persistent random walk model to describe this phenomenon. Experimentally, the authors [10] report power-law tails in the flight-length density, $p\left(x_{f}\right) \propto x_{f}^{-2}$. Figure 5 demonstrates that numerical simulations of our model are able to generate the power-law tails for flight length density and emulate the experimental data on the whole $x_{f}$ scale using reasonable parameters. Furthermore, the parameters of our new model, such as persistence probability $w$ or $\alpha$, rate $\lambda$ and speed $\nu$ can be easily found by comparing the exact analytical formula of second moment with experimental mean squared displacements.

\section{BIMODAL DENSITIES AND TRANSITION FROM DIFFUSIVE TO SUPERDIFFUSIVE REGIME.}

Surprisingly, our truncated Lévy walk model in the long time limit leads to bimodal densities in the superdiffusive regime $(1 / 2<\alpha<1)$. This phenomenon does not exist for classical superdiffusive Lévy walks. Bimodal densities (Lamperti distributions) only appear in the ballistic regime for Lévy walks with a divergent first moment for running times $[8,21,38-40]$. In the superdiffusive case, the density for Lévy walks is Gaussian in the central part with power-law tails [39]. This is completely different to densities for (10) (see Fig. 6). Density peaks for $p(x, t)$ in (10) occur at $|x|<\nu t$. Figure 6 shows the emergence of a bimodal density for $t=10^{2}$ and $w>w_{c}$, where $w_{c}=3 / 4$. The density $p(x, t)$ exhibits two distinct long time behaviors: it is Gaussian for $\alpha<1 / 2\left(w<w_{c}\right)$ and bimodal for $\alpha>1 / 2\left(w>w_{c}\right)$.

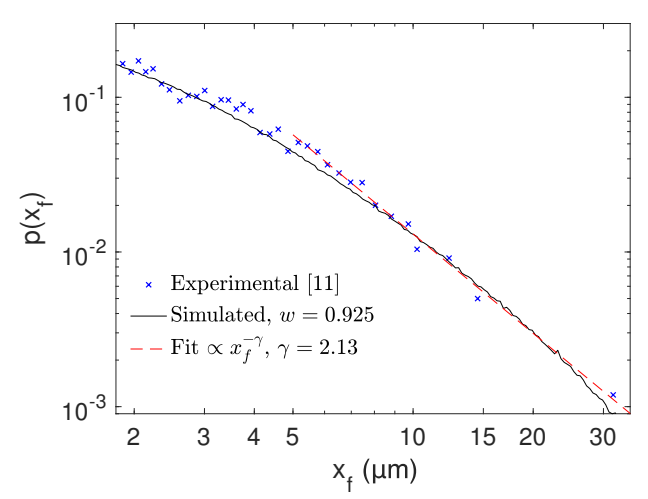

FIG. 5. The PDFs, $p\left(x_{f}\right)$, of uni-directional flight lengths, $x_{f}$ from experimental measurements (crosses) taken from [10] and simulation of underlying random walk with $w=0.925$ (solid black). Each simulation had $N=10^{5}$ particles running for $t=10^{2} \mathrm{~s}$ with $\nu=1.2 \mu \mathrm{m} \mathrm{s}^{-1}$ and $\lambda=1 \mathrm{~s}^{-1}$. The tail of the PDF was fitted with a power-law (red dashed), $p\left(x_{f}\right) \propto x_{f}^{-\gamma}$ with $\gamma=2.13$.

For $-1<\alpha<1 / 2(0<w<3 / 4)$, the variance (17) corresponds to the diffusive regime with the effective diffusion coefficient

$$
D=\frac{\nu^{2}}{\lambda(1-2 \alpha)}
$$

For alternating velocity random walks, the conventional diffusion coefficient would be $D_{0}=\nu^{2} / \lambda$. As $\alpha \rightarrow 1 / 2$, the effective diffusion coefficient tends to infinity and this indicates the transition from the diffusive to superdiffusive regime. For the long time limit of (10) in the diffusive regime when $\partial^{2} p / \partial t^{2}$ becomes negligible, the solution is Gaussian:

$$
p(x, t)=(4 \pi D t)^{-1 / 2} \exp \left(-\left(x-\mu_{\alpha} t^{\alpha}\right)^{2} / 4 D t\right),
$$

where

$$
\mu_{\alpha}=\nu(2 u-1) \lambda^{\alpha-1} / \Gamma(\alpha+1),
$$

and $D$ is defined in (20). Figure 6 shows the excellent agreement between numerical simulations and the Gaussian solution for $w=0.6$.

The bimodal distribution of $p(x, t)$ in Fig. 6 with peaks close to the maximum position $\pm \nu t$ is reminiscent of the delta function horns at $x= \pm \nu t$ ('chubchiks') in Lévy walks [39]. They too vary similarly with the parameter $\mu$, which determines the run time PDF, $\psi(t) \propto t^{-1-\mu}$. For Lévy walks in the superdiffusive case $(1<\mu<2)$, the region near the initial position is Gaussian with the tails of the distribution $|x|>\nu t$ having the distribution $p(x, t) \sim t /|x|^{1+\mu}$. Although our correlated random walk has similarities to Lévy walks, the major difference in the asymptotic density is the continuous distribution of the bimodal peaks at positions $|x|<\nu t$ instead of the chubchiks seen in Lévy walks at $|x|=\nu t$. 


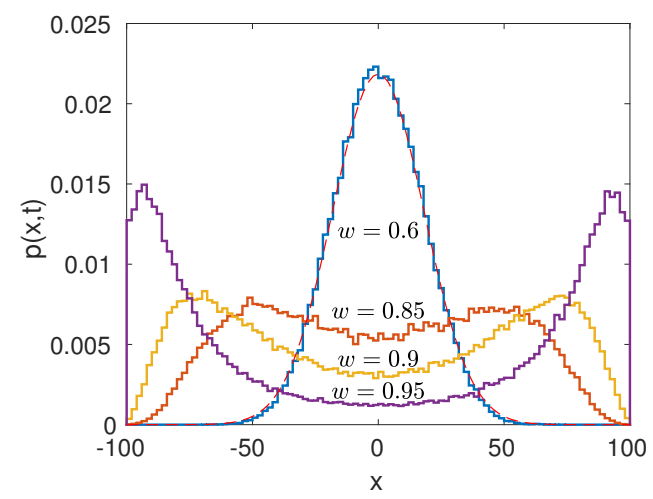

FIG. 6. The PDFs (solid lines) $p(x, t)$ for particle positions $x$ at the end of simulation time $t=10^{2}$ for varying values of $w$. The Gaussian solution (dashed line) is shown for $w=0.6$. The parameters of simulation were $N=10^{5}$ particles, $u=0.5$, $\lambda=1, \nu=1$. The initial density was $p(x, 0)=\delta(x)$.

In essence, the chubchiks of Lévy walks appear due to the group of particles that have been moving at the propagation velocity for the entire time $t$ and thus form a propagating front. Intriguingly, these fronts also appear for our correlated random walk but at very short times shown by Fig. 7. However, these propagating fronts decay exponentially with time whereas for Lévy walks they decay as $t^{1-\mu}(1<\mu<2)$. By $t=30$, the propagating front has completely 'evaporated' and the tail is now exponential with no trace of the original front. This phenomenon is intuitive since particles performing our correlated random walk take exponentially distributed runs, abeit in a persistent manner, but Lévy walks take powerlaw distributed runs. Exponential decay of the fronts can be seen in the inset of Fig. 7 where the number of particles $N(\cdot)$ with position $x>\nu t-\epsilon$ is plotted as a function of time $t$.

The evaporation of the propagating front demonstrates a non-equilibrium phase transition since the PDF shows chubchiks for short times that decay into exponential tails for long times. This shows the non-stationary nature of the random walk generated by (10) and the transition from Lévy walk like behavior at short times to a completely novel distribution for long times. Note that similar bimodal densities are observed in velocity random walks with interacting particles [41, 42].

\section{BIOLOGICAL ORIGINS}

Now, the question remains: what is the underlying biological mechanism explaining self-reinforced directionality in intracellular transport? To answer this question, we suggest a simple illustration of the possible origin for self-reinforced directionality. Let us consider endosomal motility, which is governed by the adaptor complexes on its membranes, the most notable being Rab GTPases [43]. These adaptors facilitate attachment to

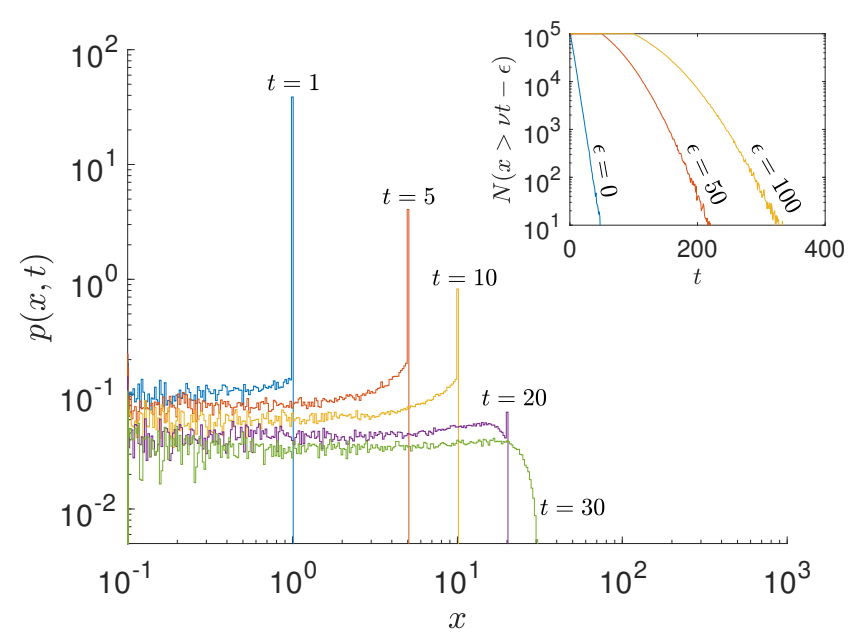

FIG. 7. Main: The PDF of particle positions, $p(x, t)$, for simulations of (9) at varying times with $w=0.8$. Other parameters are $N=10^{5}, u=0.5, \nu=1$ and $\lambda=1$ Inset: From the same simulation as the main figure we count the number of particles, $N(x>\nu t-\epsilon)$, out of $N=10^{5}$ that have position $x>\nu t-\epsilon$ with $\epsilon$ varied between 0,50 and 100. The maximum position possible is $x=\nu t$.

dynein and kinesin motors [44] and, therefore, dictate the positioning and motility of endosomes in the cell $[43,45]$. To simplify this vastly complex process, consider that the endosome contains a number, $n_{-}$, of adaptor proteins that attach to kinesin leading to transport towards the cell periphery. Similarly, $n_{+}$is the number of adaptor proteins that attach to dynein and facilitate transport towards the cell nucleus. Then, when an endosome happens to attach to a microtubule, the simplest assumption about probabilities $q_{+}$and $q_{-}$in (1) would be $q_{+}=n_{+} /\left(n_{+}+n_{-}\right)$and $q_{-}=n_{-} /\left(n_{+}+n_{-}\right)$. However, due to the complexity of endosomal transport we can introduce a weight, $w \in[0,1]$, such that $q_{ \pm}=w n_{ \pm} /\left(n_{+}+n_{-}\right)+(1-w) n_{\mp} /\left(n_{+}+n_{-}\right)$.

From the very beginning of endocytosis until degradation, endosomes undergo a maturation process, including the association of proteins, such as Rab5 and PI(3)K [46]. Recent work has show that effectors of Rab5 display distinct spatial densities [47] suggesting that $n_{-}$and $n_{+}$are functions of the time spent running towards, $t^{-}$, or away, $t^{+}$, from the cell center. So, we assume that $n_{ \pm}=n_{ \pm}^{0}+a t^{ \pm}$with $a$ being some constant rate. The more an endosome moves in towards the nucleus, the more $n_{+}$increases and vice versa. This reinforcement rule is similar to that of discrete reinforced random walks $[37,48]$. Neglecting $n_{ \pm}^{0}$, this formulation is exactly what leads to the repetition compulsion property in (2), since then $n_{-} /\left(n_{+}+n_{-}\right)=t^{-} / t$ and $n_{+} /\left(n_{+}+n_{-}\right)=t^{+} / t$. 


\section{CONCLUSION}

In this paper, we developed a persistent random walk model with finite velocity and self-reinforcing directionality that generates superdiffusion without the standard assumption of power-law distributed run times. A governing hyperbolic PDE (10) for particle probability density was derived along with exact solutions for the first and second moments. The theory is able to explain the experimentally observed self-organization of exponentially distributed runs into unidirectional flights leading to exponentially truncated Lévy walks [10]. We showed excellent agreement between the density of flight lengths from numerical simulations and in vivo cargo transport experiments. In the superdiffusive regime, numerical simula- tions of particle densities show bimodal densities (aggregation), which is a new phenomenon not seen in the classical linear hyperbolic or Lévy walk models. We believe that our methodology can be used to model migrating cancer cells [15, 49], T-cell motility [50], human foraging [13], front propagation phenomena [51], first passage time problems $[52,53]$ and viruses mobility inside cells [54].

\section{ACKNOWLEDGMENTS}

The authors acknowledge financial support from FAPESP/SPRINT Grant No. 15308-4 , EPSRC Grant No. EP/J019526/1 and the Wellcome Trust Grant No. 215189/Z/19/Z. We thank S. Granick for sharing experimental data; V. J. Allan, T. A. Waigh and P. Woodman for discussion.
[1] J. Murray, Mathematical biology: I. An introduction, vol. 17 (Springer Science \& Business Media, 2007). J. Murray, Mathematical biology II: spatial models and biomedical applications (Springer New York, 2001).

[2] A. Okubo and S. A. Levin, Diffusion and ecological problems: modern perspectives, vol. 14 (Springer Science \& Business Media, 2013).

[3] H. G. Othmer, S. R. Dunbar, and W. Alt, Journal of Mathematical Biology 26, 263 (1988).

[4] T. Hillen, Mathematical Models and Methods in Applied Sciences 12, 1007 (2002).

[5] J. Tailleur and M. Cates, Physical Review Letters 100, 218103 (2008).

[6] A. G. Thompson, J. Tailleur, M. E. Cates, and R. A. Blythe, Journal of Statistical Mechanics: Theory and Experiment 2011, P02029 (2011).

[7] V. Mendez, S. Fedotov, and W. Horsthemke, Reactiontransport systems: mesoscopic foundations, fronts, and spatial instabilities (Springer Science \& Business Media, 2010).

[8] V. Zaburdaev, S. Denisov, and J. Klafter, Reviews of Modern Physics 87, 483 (2015).

[9] A. M. Reynolds, Biology Open 7, bio030106 (2018).

[10] K. Chen, B. Wang, and S. Granick, Nature Materials 14, 589 (2015).

[11] M. S. Song, H. C. Moon, J.-H. Jeon, and H. Y. Park, Nature Communications 9, 1 (2018).

[12] S. Fedotov, N. Korabel, T. A. Waigh, D. Han, and V. J. Allan, Physical Review E 98, 042136 (2018).

N. Korabel, T. A. Waigh, S. Fedotov, and V. J. Allan, PloS One 13 (2018).

[13] D. A. Raichlen, B. M. Wood, A. D. Gordon, A. Z. Mabulla, F. W. Marlowe, and H. Pontzer, Proceedings of the National Academy of Sciences 111, 728 (2014).

[14] G. Ariel, A. Rabani, S. Benisty, J. D. Partridge, R. M. Harshey, and A. Be'er, Nature Communications 6, 8396 (2015).

[15] S. Huda, B. Weigelin, K. Wolf, K. V. Tretiakov, K. Polev, G. Wilk, M. Iwasa, F. S. Emami, J. W. Narojczyk, M. Banaszak, et al., Nature communications 9, 1 (2018).
[16] A. Compte and R. Metzler, Journal of Physics A: Mathematical and General 30, 7277 (1997).

[17] I. M. Sokolov and R. Metzler, Physical Review E 67, 010101 (2003).

[18] M. M. Meerschaert, D. A. Benson, H.-P. Scheffler, and P. Becker-Kern, Physical Review E 66, 060102 (2002).

[19] B. Baeumer, M. Meerschaert, and J. Mortensen, Proceedings of the American Mathematical Society 133, 2273 (2005).

[20] P. Becker-Kern, M. M. Meerschaert, H.-P. Scheffler, et al., The Annals of Probability 32, 730 (2004).

[21] V. Uchaikin and R. Sibatov, Journal of Physics A: Mathematical and Theoretical 44, 145501 (2011).

[22] M. Magdziarz, H.-P. Scheffler, P. Straka, and P. Zebrowski, Stochastic Processes and their Applications 125, 4021 (2015).

[23] S. Fedotov, Physical Review E 93, 020101 (2016).

[24] J. P. Taylor-King, R. Klages, S. Fedotov, and R. A. Van Gorder, Physical Review E 94, 012104 (2016).

[25] V. A. Jansen, A. Mashanova, and S. Petrovskii, Science 335, 918 (2012).

M. E. Wosniack, M. C. Santos, E. P. Raposo, G. M. Viswanathan, and M. G. da Luz, PLoS Computational Biology 13, e1005774 (2017).

[26] G. M. Viswanathan, S. V. Buldyrev, S. Havlin, M. Da Luz, E. Raposo, and H. E. Stanley, Nature 401, 911 (1999).

[27] V. Tejedor, R. Voituriez, and O. Bénichou, Physical Review Letters 108, 088103 (2012).

[28] A. Reynolds, Journal of the Royal Society Interface 9, 528 (2012).

[29] R. N. Mantegna and H. E. Stanley, Physical Review Letters 73, 2946 (1994).

[30] Á. Cartea and D. del Castillo-Negrete, Physical Review E 76, 041105 (2007).

F. Sabzikar, M. M. Meerschaert, and J. Chen, Journal of Computational Physics 293, 14 (2015).

[31] D. A. Kessler and E. Barkai, Physical Review Letters 108, 230602 (2012). 
E. Barkai, E. Aghion, and D. Kessler, Physical Review X 4, 021036 (2014).

[32] S. Fedotov, G. Milstein, and M. Tretyakov, Journal of Physics A: Mathematical and Theoretical 40, 5769 (2007).

[33] D. R. Cox and H. D. Miller, The theory of stochastic processes (Chapman and Hall, 1965).

[34] M. J. Schnitzer, Physical Review E 48, 2553 (1993).

[35] M. Kac, The Rocky Mountain Journal of Mathematics 4, 497 (1974).

[36] G. M. Schütz and S. Trimper, Physical Review E 70, 045101 (2004).

F. N. Paraan and J. Esguerra, Physical Review E 74, 032101 (2006).

M. Da Silva, J. C. Cressoni, G. M. Schütz, G. Viswanathan, and S. Trimper, Physical Review E 88, 022115 (2013).

M. da Silva, E. Rocha, J. Cressoni, L. da Silva, and G. Viswanathan, Physica A: Statistical Mechanics and its Applications 538, 122793 (2020).

[37] T. Hillen and A. Stevens, Nonlinear Analysis: Real World Applications 1, 409 (2000).

A. Stevens and H. G. Othmer, SIAM Journal on Applied Mathematics 57, 1044 (1997).

[38] V. Uchaikin and R. Sibatov, Journal of Experimental and Theoretical Physics 109, 537 (2009).

[39] J. Klafter and I. M. Sokolov, First steps in random walks: from tools to applications (Oxford University Press, 2011).

[40] D. Froemberg, M. Schmiedeberg, E. Barkai, and V. Zaburdaev, Physical Review E 91, 022131 (2015).

[41] F. Lutscher, A. Stevens, et al., Journal of Nonlinear Science 12, 619 (2002).

R. C. Fetecau and R. Eftimie, Journal of mathematical biology 61, 545 (2010).

J. A. Carrillo, R. Eftimie, and F. K. Hoffmann, Kinetic \& Related Models 8, 413 (2015).
[42] S. Fedotov and N. Korabel, Physical Review E 95, 030107 (2017).

[43] H. Stenmark, Nat. Rev. Mol. Cell Biol. 10, 513 (2009).

[44] L. Urnavicius, C. K. Lau, M. M. Elshenawy, E. MoralesRios, C. Motz, A. Yildiz, and A. P. Carter, Nature 554, 202 (2018).

[45] J. Gindhart and K. Weber (2009).

[46] J. Huotari and A. Helenius, The EMBO Journal 30, 3481 (2011).

[47] R. Villaseñor, Y. Kalaidzidis, and M. Zerial, Current Opinion in Cell Biology 39, 53 (2016).

[48] B. Davis, Probability Theory and Related Fields 84, 203 (1990).

[49] C. T. Mierke, B. Frey, M. Fellner, M. Herrmann, and B. Fabry, Journal of Cell Science 124, 369 (2011).

C. T. Mierke, New Journal of Physics 15, 015003 (2013).

[50] T. H. Harris, E. J. Banigan, D. A. Christian, C. Konradt, E. D. T. Wojno, K. Norose, E. H. Wilson, B. John, W. Weninger, A. D. Luster, et al., Nature 486, 545 (2012).

[51] V. Méndez, D. Campos, and S. Fedotov, Physical Review E 70, 036121 (2004).

S. Fedotov, A. Tan, and A. Zubarev, Physical Review E 91, 042124 (2015).

[52] S. Redner, A guide to first-passage processes (Cambridge University Press, 2001).

[53] S. Condamin, O. Bénichou, V. Tejedor, R. Voituriez, and J. Klafter, Nature 450, 77 (2007).

L. Angelani, R. Di Leonardo, and M. Paoluzzi, The European Physical Journal E 37, 59 (2014).

[54] T. Lagache and D. Holcman, SIAM Journal on Applied Mathematics 68, 1146 (2008).

U. F. Greber and M. Way, Cell 124, 741 (2006).

M. P. Dodding and M. Way, The EMBO Journal 30, 3527 (2011). 